\title{
Relation Between the Prevalence of Diabetes Mellitus and Human Development Index: A Global Ecological Study
}

\author{
Salman Khazaei, ${ }^{1}$ Shahab Rezaeian, ${ }^{2}$ Somayeh Khazaei, ${ }^{3}$ and Shahrzad Nematollahi ${ }^{4,}$ \\ ${ }^{1}$ Department of Epidemiology and Biostatistics, School of Public Health, Hamadan University of Medical Sciences, Hamadan, IR Iran \\ ${ }^{2}$ Kurdistan Research Center for Social Determinants of Health, Kurdistan University of Medical Sciences, Sanandaj, IR Iran \\ ${ }^{3}$ Department of Operating Room, Rafsanjan University of Medical Sciences, Rafsanjan, IR Iran \\ ${ }^{4}$ Department of Epidemiology and Biostatistics, School of Public Health, Tehran University of Medical Sciences, Tehran, IR Iran \\ "Corresponding author: Shahrzad Nematollahi, Department of Epidemiology and Biostatistics, School of Public Health, Tehran University of Medical Sciences, Tehran, IR Iran. \\ Tel: +98-9136560762, E-mail: shahrzadnema@yahoo.com
}

Received 2016 June 20; Revised 2016 August 28; Accepted 2016 September 17.

\begin{abstract}
Background: Diabetes mellitus (DM) is one of the major causes of morbidity and mortality worldwide, but little is known about the effect of Human Development Index (HDI) on the prevalence rate of DM.

Objectives: This study aimed to identify the variations in DM-specific rates by HDI among world countries.

Methods: This global ecological study was performed based on the data from the international diabetes federation (IDFD) and the world bank report for the year 2013. The analysis for this study was performed on ecological data available for 161 countries in 2013. Results: Globally, the prevalence of DM ranged between 4\% and12\% in 2013, with the highest prevalence in Middle-East and Northern African $(>12 \%$ ) and the lowest in Sub-Saharan countries (<4\%). The prevalence of DM was high in older age groups in both low-to middle, and high income regions. In very-high or high HDI regions men were more likely to have diabetes, whereas in low HDI region women were more affected. The prevalence ranges of DM were 7\% - 12\%, 5\% - 7\%, 5\% - 9\%, and 4\% - 12\% among high-income, low-to-middle income, low-income, and upper-middle income countries, respectively. The rate of diabetes comparative prevalence, incidence of diabetes type-I, and impaired glucose tolerance comparative prevalence were 7.2\%, 22.7 (per 100,000 people aged 0-14 years), and 8.3\% in very high and 8.7\%, 16.2 (per 100,000 people aged 0 - 14 years), and 8.7\% in high HDI regions, respectively.

Conclusions: The pandemic of diabetes is a major public health problem around the world, mostly in the countries with high and very high HDI. In addition, the prevalence of diabetes is associated with population pyramid and the income level of countries. Knowledge of national and regional rates of diabetes and associated factors can help policymakers to optimize diabetes control in countries with limited resources.
\end{abstract}

Keywords: Diabetes Mellitus, Human Development Index, Ecological Study

\section{Background}

Diabetes mellitus (DM) is one of the major causes of morbidity and mortality worldwide, causing a huge burden of illness and impairments of the quality of life among affected people (1). It is associated with serious and longterm complications such as blindness, amputation, renal disease and failure, circulatory disorders, impaired quality of life, and increased use of health services $(2,3)$.

There was a global estimate of 285 billion adults with DM in 2010 (4), and the prevalence of DM for all age-groups is estimated to be $4.4 \%$ worldwide in 2030 (5). The prevalence of DM will globally continue to increase by $30 \%$, which equals 300 million new diabetic adults in 2025 (6). The epidemic of DM type II is growing in parallel with rapid urbanization, nutrition transition, and sedentary lifestyle (7). With more than $50 \%$ of the world population are now urban dwellers, mostly in developing world, current evidence suggest an association between urban living arrangements and chronic diseases such as DM (5, 6, 8-10).
This situation even worsens in high density countries such as China and India where the DM prevalence could be as high as 2.5 times in urban areas than that in rural areas (11).

The epidemic of DM has been attributed to the epidemiological transitions, global trends away from traditional lifestyles and towards urbanization. Having a great impact of urbanization and migrations on affluence of the communities, the highest rates of DM have been observed in newly well-off populations $(12,13)$. In addition, urbanization and migration accelerate the prevalence of some factors, which are widely accepted as risk factors for DM such as obesity, nutrition transition, and physical inactivity (14-17). In many non-industrialized populations, obesity is regarded as a symbol of health and wealth, which makes prevention even more complicated (18). Some authors suggested that the transition is consistently increased with the gross national product per capita of a country (18-20).

Having investigated several factors associated with DM, unequal distribution of the human development in- 
dex (HDI) has not been investigated yet. the human development index is comprised of three components namely education, life expectancy and gross national income (GNI) with ranges between 0 to 1 , and is appropriate for defining socio-economic status of the societies (21). Diabetes mellitus has become a prominent cause of morbidity and mortality all around the world $(2,22)$, necessitating the tendency towards incorporation of the socio-economic status into diagnostic measures.

Due to lack of data for many countries, data have to be extrapolated from probably dissimilar countries; therefore, further epidemiological investigation in every region is urgently needed (23). Good-quality data on disease burden are crucial to aid planning and implementation of prevention and control strategies for diabetes.

\section{Objectives}

Thus, this study aimed to identify the variations in DMspecific rates by HDI among countries worldwide.

\section{Methods}

This is an ecological study on the relationship between the prevalence of DM and the HDI. Data for the prevalence of DM from countries in 2013 was obtained from the international diabetes federation (IDF).

The IDF is an umbrella organization of over 230 national diabetes associations in 170 countries and territories. The IDF is divided into seven regions, with the aim of strengthening the work of national diabetes associations and enhancing the collaboration between them. Available diabetes data for countries in IDF include diabetes national and comparative prevalence (\%), incidence type 1 diabetes ( 0 - 14 yr.) per 100,000, IGT national and comparative prevalence (\%), number of people with diabetes by sex and different age groups, and number of people with undiagnosed diabetes (20 - 79 yr.) in $1000 \mathrm{~s}$ by country. Data about the HDI and other indices were obtained from the World Bank report 2013 available at http://databank.worldbank.org/data/reports.aspx. The Human Development Index has several main components that include life expectancy at birth, mean years of schooling, and gross national income (GNI) per capita. The analysis was restricted to countries for which both the epidemiologic data from DM and HDI were available (161 countries). All countries were stratified into four categories by HDI. Comparisons among HDI categories (very high, high, medium, and low) were done with One-way ANOVA. The prevalence rates of diabetes types I and II were calculated for each region based on HDI, while we considered different covariates such as gender, residency, life expectancy at birth (LEB), mean years of schooling (MYS), and GNI. Moreover, the diabetes comparative prevalence (DCP), incidence of diabetes type I (IDTI), and impaired glucose tolerance comparative prevalence (IGTCP) for each HDI category were calculated. The national or regional prevalence indicates the percentage of each country's or region's population that has diabetes. The comparative prevalence has been calculated by assuming that every country and region has the same age profile (the age profile of the world population has been used). This removes the differences of age between countries and regions, and makes this figure ideal for making comparisons.

Statistical tests and visual demonstrations were used to present the results. Correlation bivariate methods were used for assessment of the correlation between the DM prevalence rate and the HDI. All analyses were conducted at 95\% significant level using the Stata software version 12 (StataCorp, College Station, Texas, USA).

\section{Results}

Overall 161 countries were included in the study. All the available data from World Bank for 175 countries were used. Figure 1 shows the global estimates of diabetes mellitus prevalence in 2013. Accordingly, Middle-East Asian countries such as Saudi Arabia and Turkey; and Northern African countries such as Sudan and Egypt had the highest prevalence of diabetes ( $>12 \%)$. The lowest prevalence was reported from Sub-Saharan countries such as Mali, Niger, Nigeria; countries of Southern Africa such as Angola, Botswana, Madagascar; and Eastern Europe such as Belarus and Ukraine $(<4 \%)$. Overall, most of the countries had a diabetes prevalence ranged between $4 \%$ and $9 \%$.

Categorizing the countries into four distinct categories based on an income level; the highest prevalence of diabetes among high-income group was in Saudi Arabia and Northern American (7\% - 12\%); among the low-tomiddle income group was in Iraq, Egypt, and Sudan, India, and Mongolia (5\% - 7\%); among the low-income group was in South-East of Africa such as Ethiopia, Somalia, Democratic Republic of Congo, Tanzania, and Chad (5\% - 9\%), and among upper -middle income countries was in Mexico, Brazil, Colombia, Peru, and Argentina (4\% - 9\%) and in China, Kazakhstan, Iran, Turkey, Libya, and Algeria (9\% $-12 \%$ ) (Figure 2).

Figure 3 shows the prevalence of diabetes in different HDI regions by age group in 2013. The highest and lowest prevalence rates of diabetes were in 40-59 and 60 - 79 years old in low or middle HDI, and 60 - 79 and 20 - 39 years in high or very-high HDI countries, respectively.

Based on the HDI categories, we compared the highest prevalence of diabetes according to gender and resi- 


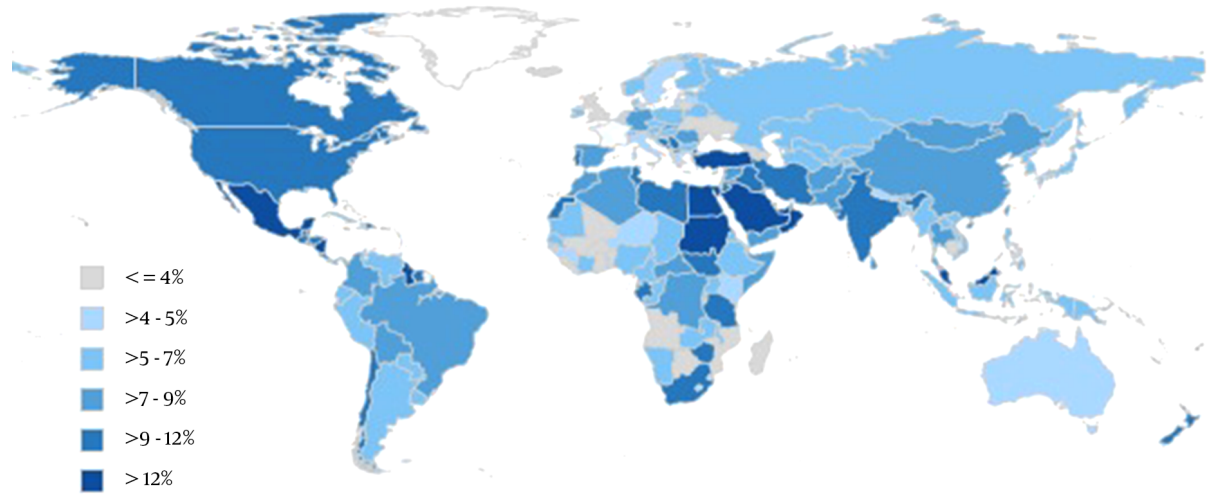

Figure 1. Estimated Prevalence of Diabetes Worldwide in 2013

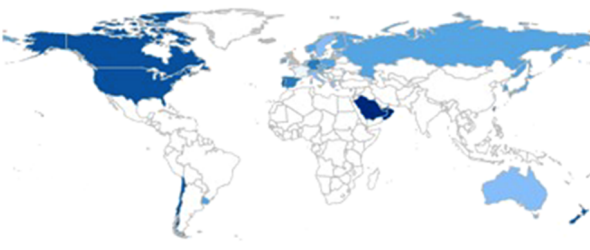

High Income Countries

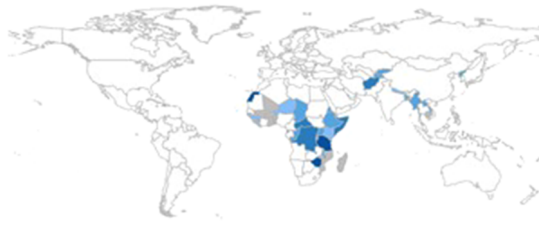

Low Income Countries

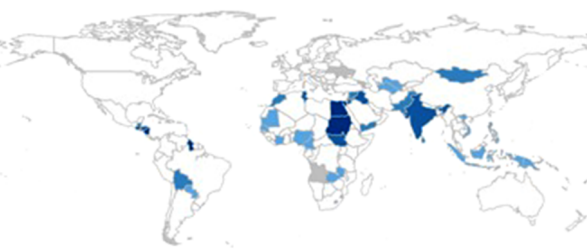

Low and Middle Income Countries

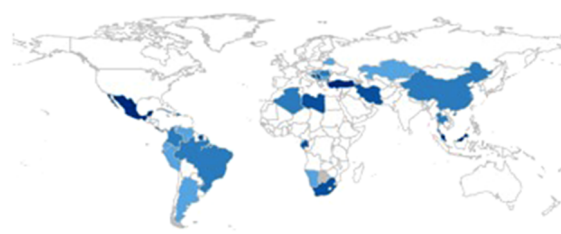

Upper Middle Income Countries

Figure 2. Estimated Prevalence of Diabetes by Income Status Worldwide in 2013

dency (Table 1). In very-high or high HDI regions, men were more likely to have diabetes, whereas in low HDI regions women were more affected. There was only one category, namely the middle HDI region, where the proportion of men and women affected by diabetes was almost the same ( $5 \%$ versus $4.8 \%$ ). Finally, we calculated some indices according to HDI regions. Table 2 demonstrates that the highest percentages of DCP, IDTI, and IGTCP were occurred in high, very-high, and high HDI regions, respectively. Figure 4 demonstrates a positive significant correlation between the HDI and DCP $(\mathrm{P}<0.001)$.

\section{Discussion}

The prevalence of DM globally ranged between $4 \%$ and $12 \%$ in 2013 , and the prevalence ranges were $7 \%-12 \%, 5 \%$ 7\%, 5-9\%, and 4\% - 12\% among high-income, low-to-middle income, low-income, and upper-middle income countries, respectively. We found a positive significant correlation between the HDI and DCP.

Despite WHO emphasis on the urgent need to action, magnitude and seriousness of DM are still unrecognized in many regions. As a consequence, increasing prevalence of the disease and the long-term cost of therapy for both patients and the health sectors are prizes to pay for this ig- 

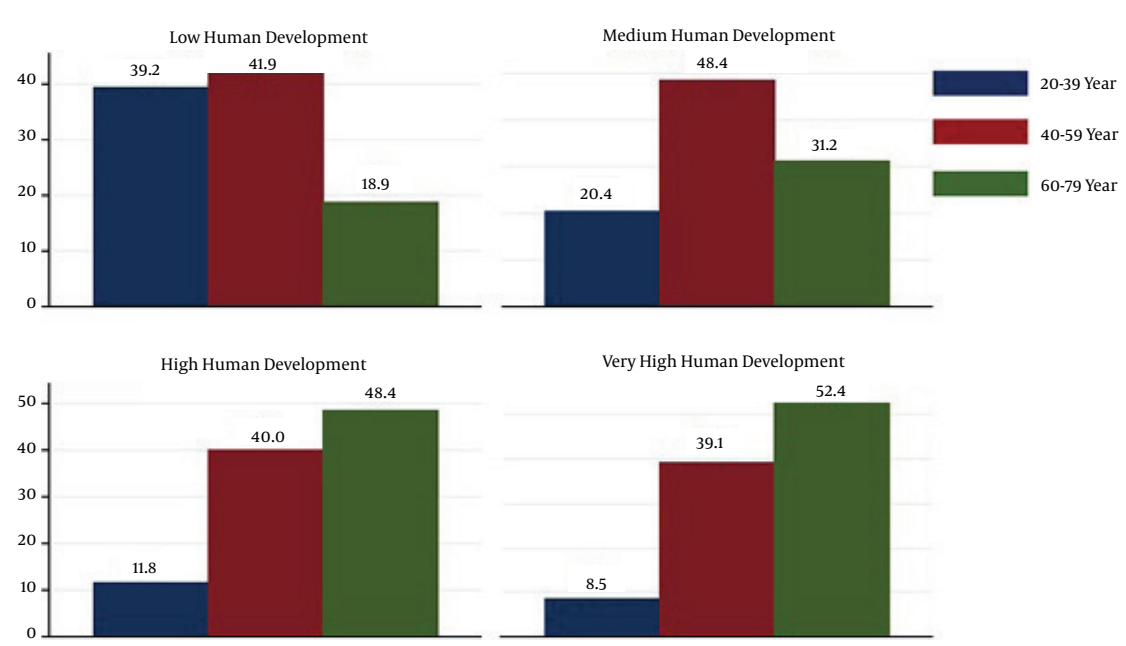

Figure 3. Prevalence of Diabetes in Different HDI Regions by Age Group in 2013

Table 1. Prevalence of Diabetes (\%) Stratified by Gender and Residency in Different HDI Regions in 2013

\begin{tabular}{lcccc}
\hline \multirow{2}{*}{ Region } & \multicolumn{2}{c}{ Gender } & \multicolumn{2}{c}{ Residency } \\
\cline { 2 - 5 } & Men Mean $(\mathbf{C I})$ & Women Mean $(\mathbf{C I})$ & Urban Mean $(\mathrm{CI})$ & Rural Mean $(\mathbf{C I})$ \\
\hline Very high human development (11 countries) & $9.8(8.4,11.1)$ & $8.6(7.1,10.1)$ & $9.3(7.9,10.7)$ & $8.4(6.9,9.8)$ \\
High human development (33 countries) & $10.3(8.4,12.2)$ & $8.3(7.3,9.2)$ & $9.7(8.3,11.1)$ & $7.3(6.7,7.9)$ \\
\hline Medium human development (88 countries) & $7.1(6.4,7.8)$ & $8.0(7.0,9.0)$ & $8.8(7.9,9.7)$ & $6.5(5.7,7.2)$ \\
\hline Low human development (29 countries) & $5.0(4.1,5.9)$ & $4.8(3.9,5.6)$ & $6.7(5.6,7.8)$ & $4.1(3.3,4.9)$ \\
World (161 countries) & $8.7(8,9.5)$ & $9.1(8.3,9.8)$ & $8.6(8.0,9.2)$ & $6.3(5.8,6.8)$ \\
\hline
\end{tabular}

Abbreviation: $\mathrm{CI}$, confidence interval.

Table 2. Diabetes Status According to HDI Component Worldwide in 2013

\begin{tabular}{|c|c|c|c|c|c|c|c|}
\hline \multirow[t]{2}{*}{ Region } & \multicolumn{3}{|c|}{ Diabetes Status } & \multicolumn{4}{|c|}{ HDI Component } \\
\hline & DCP Mean (CI) & IDT1 Mean $(\mathrm{CI})$ & IGTCP Mean (CI) & LEB & MYS & GNI & HDI \\
\hline Very high human development & $7.2(6.1,8.4)$ & $22.7(16,28.6)$ & $8.3(6.6,10.1)$ & 80.2 & 11.7 & 40,046 & 0.89 \\
\hline High human development & $8.7(6.6,10.7)$ & $16.2(12.5,19.9)$ & $8.7(7.3,10.2)$ & 74.5 & 8.1 & 13,231 & 0.74 \\
\hline Medium human development & $8.1(7.3,8.9)$ & $5.1(3.6,6.6)$ & $8.4(7.9,9)$ & 67.9 & 5.5 & 5,960 & 0.61 \\
\hline Low human development & $5.6(4.6,6.6)$ & $2.8(1.8,7.6)$ & $7.7(7.4,8.1)$ & 59.4 & 4.2 & 2,904 & 0.49 \\
\hline Pvalue (F-test) & $<0.001$ & $<0.001$ & $<0.001$ & $<0.001$ & $<0.001$ & $<0.001$ & - \\
\hline
\end{tabular}

Abbreviations: DCP, diabetes comparative prevalence (\%); IDT1, incidence type 1 diabetes (0 - 14 year) per 100,000; IGTCP, IGT comparative prevalence (\%); HDI, human development index; LEB, life expectancy at birth; MYS, mean years of schooling; GNI, gross national income per capita; CI, confidence interval.

norance (24).

We found that Middle-East Asian countries such as Saudi Arabia and Turkey; and Northern African countries such as Sudan and Egypt had the highest prevalence of diabetes. These findings can be attributed to previous reports in many developing regions, specifically Mexico, Egypt, and South Africa, regarding exceeding prevalence of obesity compared to developed countries. There is further evidence that the rates of increase in obesity among adults in Asia, North Africa, and Latin America are 2-5 times of the rate of increase in Northern America (25).

Currently, Asia accounts for $60 \%$ of the world's dia- 


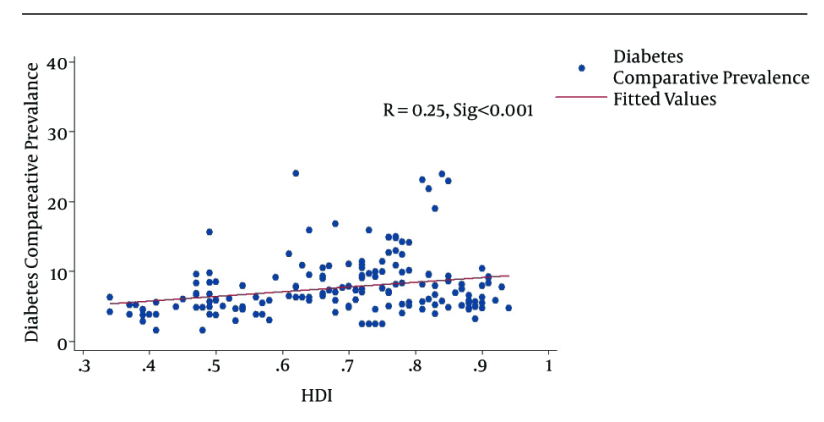

Figure 4. Correlation Between the Human Development Index and Diabetes Comparative Prevalence

betic population. Compared with Western populations, Asians develop diabetes, at lower body weights, and at much higher rates given the same amount of weight gain $(26,27)$.

Possible factors contribute to accelerated diabetes epidemic in Asian countries can be described as the "normalweight metabolically obese" phenotype, high prevalence of smoking and alcohol use, high intake of refined carbohydrates, and dramatic decrease of physical activity levels (7).

We also showed dependency of the age-specific DM prevalence on the development level of countries. A study to determine the effect of income on type 2 diabetes showed that after controlling for age, having a household income of $\$ 29,999$ per year was associated with the type 2 diabetes prevalence (28). In addition, DM was regarded as a health problem in middle-aged living in low or medium developed countries, and elderlies living in high developed countries. This finding is acknowledged by previous studies reporting that unlike industrialized countries, where the highest number of people with diabetes will be in the oldest age groups. Diabetes Mellitus is a health problem of 45- 64 years old in developing countries such as Asia $(6,29,30)$. Many of the large number of people becoming diabetic in middle age will experience its chronic complications during their working lives. Therefore, prevention of diabetes in developing world has a huge impact on future therapeutic costs (31).

Diabetes has several specific characteristics: (i) its common risk factors (unhealthy diets, physical inactivity and harmful alcohol use) are potentially amenable to behavioral modification; (ii) it is detectable using simple tests and managed in primary-care settings in low-income countries (32); and (iii) it is the focus of efforts to ensure greater prioritization of non-communicable diseases (NCDs) on the global research agenda (23). Translating these findings into practice, however, requires fundamental changes in public policies, and health systems (7).
Appropriate glycemic control requires that the patient perceives the disease. Poverty and lower levels of education in non-industrialized countries are potential obstacles for that perception (33). Therefore, curbing the escalating diabetes epidemic, primary prevention through promotion of a healthy diet and lifestyle should be a global public policy priority regardless of the level of county income or development (7).

Our study had several limitations. In this ecological study, the global data from 161 countries were used. Hence, the aggregated data could not be any individual-level interpretation and may cause the ecological fallacy. In addition, we had no information about other factors affecting diabetes such as nutrition, which this factor varies among and between countries. This issue may affect the result and the findings should be warily interpreted. Despite the limitations, our results provide important evidence on the relationship between DM and HDI worldwide.

\subsection{Conclusions}

The pandemic of diabetes is a major public health problem around the world, mostly in the countries with high and very high HDI. In addition, the prevalence of diabetes is associated with population pyramid and the income level of countries. Knowledge of national and regional rates of diabetes and associated factors can help policymakers to optimize diabetes control in countries with limited resources.

\section{Acknowledgments}

We used the data from the international diabetes federation (IDFD) and the World Bank report for this study. This research has not received any specific fund. The authors declare that they have no conflict of interests.

\section{Footnote}

Authors' Contribution: All authors contributed equally and participated in the data extraction, analysis and interpretation. All authors critically reviewed, refined and approved the manuscript.

\section{References}

1. Hakkinen A, Kukka A, Onatsu T, Jarvenpaa S, Heinonen A, Kyrolainen $\mathrm{H}$, et al. Health-related quality of life and physical activity in persons at high risk for type 2 diabetes. Disabil Rehabil. 2009;31(10):799-805. doi: 10.1080/08916930802354930. [PubMed: 19034723].

2. Sikdar KC, Wang PP, MacDonald D, Gadag VG. Diabetes and its impact on health-related quality of life: a life table analysis. Qual Life Res. 2010;19(6):781-7. doi: 10.1007/s11136-010-9641-5. [PubMed: 20349211]. 
3. Zhang P, Zhang X, Brown J, Vistisen D, Sicree R, Shaw J, et al. Global healthcare expenditure on diabetes for 2010 and 2030. Diabetes Res Clin Pract. 2010;87(3):293-301. doi: 10.1016/j.diabres.2010.01.026. [PubMed: 20171754].

4. Shaw JE, Sicree RA, Zimmet PZ. Global estimates of the prevalence of diabetes for 2010 and 2030. Diabetes Res Clin Pract. 2010;87(1):4-14. doi: 10.1016/j.diabres.2009.10.007. [PubMed:19896746].

5. Wild S, Roglic G, Green A, Sicree R, King H. Global prevalence of diabetes: estimates for the year 2000 and projections for 2030. Diabetes Care. 2004;27(5):1047-53. [PubMed: 15111519].

6. King H, Aubert RE, Herman WH. Global burden of diabetes, 19952025: prevalence, numerical estimates, and projections. Diabetes Care. 1998;21(9):1414-31. [PubMed: 9727886].

7. Hu FB. Globalization of diabetes: the role of diet, lifestyle, and genes. Diabetes Care. 2011;34(6):1249-57. doi: 10.2337/dc11-0442. [PubMed: 21617109].

8. Rivera JA, Barquera S, Gonzalez-Cossio T, Olaiz G, Sepulveda J. Nutrition transition in Mexico and in other Latin American countries. Nutr Rev. 2004;62(7 Pt 2):149-57. [PubMed: 15387482].

9. Shetty PS. Nutrition transition in India. Public Health Nutr. 2002;5(1A):175-82. doi: 10.1079/PHN2001291. [PubMed: 12027282].

10. Maletnlema TN. A Tanzanian perspective on the nutrition transition and its implications for health. Public Health Nutr. 2002;5(1A):163-8. [PubMed: 12027280].

11. Mohan V, Mathur P, Deepa R, Deepa M, Shukla DK, Menon GR, et al. Urban rural differences in prevalence of self-reported diabetes in Indiathe WHO-ICMR Indian NCD risk factor surveillance. Diabetes Res Clin Pract. 2008;80(1):159-68. doi: 10.1016/j.diabres.2007.11.018. [PubMed: 18237817].

12. Fall $\mathrm{CH}$. Non-industrialised countries and affluence. $\mathrm{Br}$ Med Bull. 2001;60:33-50. [PubMed: 11809617].

13. Herman WH, Ali MA, Aubert RE, Engelgau MM, Kenny SJ, Gunter EW, et al. Diabetes mellitus in Egypt: risk factors and prevalence. Diabet Med. 1995;12(12):1126-31. [PubMed: 8750225].

14. Cockram CS. Diabetes mellitus: perspective from the Asia-Pacific region. Diabetes Res Clin Pract. 2000;50 Suppl 2:3-7. [PubMed:11024577].

15. Passos VM, Barreto SM, Diniz LM, Lima-Costa MF. Type 2 diabetes: prevalence and associated factors in a Brazilian community-the Bambui health and aging study. Sao Paulo Med J. 2005;123(2):66-71. [PubMed: 15947833].

16. Astrup A, Dyerberg J, Selleck M, Stender S. Nutrition transition and its relationship to the development of obesity and related chronic diseases. Obes Rev. 2008;9 Suppl 1:48-52. doi: 10.1111/j.1467789X.2007.00438.x. [PubMed: 18307699].

17. Allender S, Wickramasinghe K, Goldacre M, Matthews D, Katulanda P. Quantifying Urbanization as a Risk Factor for Noncommunicable Disease.J Urban Health-Bulletin New York Acad Med. 2011;88(5):906-18. doi: 10.1007/s11524-011-9586-1.

18. Drewnowski A, Popkin BM. The nutrition transition: new trends in the global diet. Nutr Rev. 1997;55(2):31-43. [PubMed: 9155216].

19. Huisman M, Kunst AE, Bopp M, Borgan JK, Borrell C, Costa G, et al. Educational inequalities in cause-specific mortality in middle-aged and older men and women in eight western European populations. Lancet. 2005;365(9458):493-500. doi: 10.1016/S0140-6736(05)17867-2.
[PubMed: 15705459].

20. Ezzati M, Vander Hoorn S, Lawes CM, Leach R, James WP, Lopez AD, et al. Rethinking the "diseases of affluence" paradigm: global patterns of nutritional risks in relation to economic development. PLoS Med. 2005;2(5):133. doi: 10.1371/journal.pmed.0020133. [PubMed: 15916467]

21. Alkire S. Human Development: Definitions, Critiques, and Related Concepts, Background paper for the 2010. UK: Human Development Report; 2010.

22. Strong K, Mathers C, Leeder S, Beaglehole R. Preventing chronic diseases: how many lives can we save? Lancet. 2005;366(9496):1578-82. doi: 10.1016/S0140-6736(05)67341-2. [PubMed: 16257345].

23. Maher D, Waswa L, Baisley K, Karabarinde A, Unwin N, Grosskurth H. Distribution of hyperglycaemia and related cardiovascular disease risk factors in low-income countries: a cross-sectional populationbased survey in rural Uganda. Int J Epidemiol. 2011;40(1):160-71. doi 10.1093/ije/dyq156. [PubMed: 20926371].

24. Abdulganiyu G, Fola T. What is the cost of illness of type II Diabetes Mellitus in a Developing Economy? Int J Pharmacy Pharmaut Sci. 2014;6(2):927-31.

25. Popkin BM, Gordon-Larsen P. The nutrition transition: worldwide obesity dynamics and their determinants. Int $J$ Obes Relat Metab Disord. 2004;28 Suppl 3:2-9. doi: 10.1038/sj.ijo.0802804. [PubMed: 15543214].

26. Chan JC, Malik V, Jia W, Kadowaki T, Yajnik CS, Yoon KH, et al. Diabetes in Asia: epidemiology, risk factors, and pathophysiology. JAMA. 2009;301(20):2129-40. doi: 10.1001/jama.2009.726. [PubMed 19470990].

27. Yoon KH, Lee JH, Kim JW, Cho JH, Choi YH, Ko SH, et al. Epidemic obesity and type 2 diabetes in Asia. Lancet. 2006;368(9548):1681-8. doi: 10.1016/S0140-6736(06)69703-1. [PubMed: 17098087].

28. Bird $Y$, Lemstra $M$, Rogers $M$, Moraros J. The relationship between socioeconomic status/income and prevalence of diabetes and associated conditions: A cross-sectional population-based study in Saskatchewan, Canada. Int J Equity Health. 2015;14:93. doi: 10.1186/s12939-015-0237-0. [PubMed: 26458543].

29. Amos AF, McCarty DJ, Zimmet P. The rising global burden of diabetes and its complications: estimates and projections to the year 2010. Diabet Med. 1997;14 Suppl 5:1-85. [PubMed: 9450510].

30. Murray CJL, Lopez AD. The Global Burden of Disease: A Com prehensive Assessment of Mortality and Disability from Diseases, Injuries, and Risk Factors in 1990 and Projected to 2020 World Health Organisation: Geneva; 1996. Available from: http://webcache. googleusercontent.com.

31. Chugh KS, Kumar R, Sakhuja V, Pereira BJ, Gupta A. Nephropathy in type 2 diabetes mellitus in Third World countries-Chandigarh study. Int J Artif Organs. 1989;12(5):299-302. [PubMed: 2786852].

32. Beaglehole R, Epping-Jordan J, Patel V, Chopra M, Ebrahim S, Kidd $\mathrm{M}$, et al. Improving the prevention and management of chronic disease in low-income and middle-income countries: a priority for primary health care. Lancet. 2008;372(9642):940-9. doi: 10.1016/S0140 6736(08)61404-X. [PubMed: 18790317].

33. Gulliford MC. Controlling non-insulin-dependent diabetes mellitus in developing countries. Int J Epidemiol. 1995;24 Suppl 1:53-9. [PubMed: 7558552]. 